\title{
Validation of segmental visual scar quantification in ischemic and non-ischemic cardiomyopathy: Comparison to signal threshold analysis of delayed enhancement MR images Nowell Fine*1, Matthew Brymer ${ }^{2}$, Anna MacDonald ${ }^{3}$, Maria Drangova ${ }^{2}$ and James White 3
}

Address: ${ }^{1}$ London Health Sciences Centre, London, ON, Canada, ${ }^{2}$ Robarts Research Institute, London, ON, Canada and ${ }^{3}$ London Health Sciences Centre/Robarts Research Institute/Lawson Health Research Institute, London, ON, Canada

* Corresponding author

from I3th Annual SCMR Scientific Sessions

Phoenix, AZ, USA. 21-24 January 2010

Published: 21 January 2010

Journal of Cardiovascular Magnetic Resonance 2010, I 2(SuppI I):PI69 doi:10.I I86/I532-429X-I2-SI-PI69

This abstract is available from: http://jcmr-online.com/content/I2/SI/PI69

(c) 2010 Fine et al; licensee BioMed Central Ltd.

\section{Objective}

To determine the correlation between quantification of myocardial scar volume using segmental visual scoring and semi-automated signal threshold techniques.

\section{Background}

Visual evaluation of delayed enhancement (DE) MR images using a 5-point, 17-segment model is efficient and more practical than computerized quantitative signal analysis techniques. However, the accuracy of this technique for quantifying scar burden has not been validated.

\section{Methods}

161 consecutive patients with abnormal DE findings were included. Blinded visual quantification of segmental (AHA 17-segment model) scar was performed using a 5point scale, as follows; $0=$ none, $1=1-25 \%, 2=26-50 \%$, $3=51-75 \%, 4=76-100 \%$. Each segment was assumed to represent $1 / 17^{\text {th }}$ of the LV mass. Computerized, signal threshold-based analysis of total myocardial scar volume was also performed using cut-offs of 2, 3 and 5 SD above mean normal (nulled) myocardial signal. Comparison of visual scar quantification and signal-threshold based quantification was then performed using linear regression. Bland-Altman plots were then performed between visual scar volume and threshold-based scar volume measurements.

\section{Results}

Of 161 patients studied, the mean age was $57.5 \pm 13.0$ years. The mean LV ejection fraction was $46.3 \pm 20.6 \%$. Sixty-six (41\%) patients had an ischemic DE pattern, 76 $(47 \%)$ had a non-ischemic DE pattern, and $19(12 \%)$ had a combined pattern. The Pearson correlation coefficients for visual versus threshold-based quantitative analysis at 5,3 , and 2 SD signal intensity thresholds were $r=0.81, r$ $=0.79$ and $r=0.63(p<0.0001)$, respectively. Coefficients for patient sub-groups with ischemic and non-ischemic DE were $\mathrm{r}=0.81, \mathrm{r}=0.81, \mathrm{r}=0.74(\mathrm{p}<0.0001)$ and $\mathrm{r}=$ $0.72, r=0.69, r=0.46(\mathrm{p}=0.0001)$, respectively. BlandAltman analysis of the mean difference in total scar volume between visual and threshold-based analysis (5 SD) for all patients, patients with ischemic $\mathrm{DE}$, and patients with non-ischemic DE was $4.3 \pm 7.9 \%, 4.8 \pm 7.8$, and 2.6 $\pm 7.6 \%$, respectively.

\section{Conclusion}

Visual quantification of myocardial scar using a 5-point, 17-segment approach correlates well with thresholdbased quantitative analysis. In this study the enhanced volume by visual scoring was closely related to both 3 and 5 SD signal-based analysis for both ischemic and nonischemic patterns of DE. 